\title{
Development and Conservation of Basic Educational Skills in Individuals with Down Syndrome: A Longitudinal Research Study
}

\author{
A. Luigi Sangalli ${ }^{1}$, Angelo Lascioli $^{2}$, Andrea lascioli $^{3}$ \\ ${ }^{1}$ University of Verona, ${ }^{2}$ University of Verona, ${ }^{3}$ Expert in the assessment and treatment of \\ learning disabilities \\ Italy
}

\begin{abstract}
We report the first results of a longitudinal research carried out on a sample of 100 people with Down syndrome, with the aim of verifying the skills achieved, maintained and preserved in reading and writing, over a period of time. The aim of this research is to rebuild the rehabilitation path of each subject through a structured interview questionnaire. At present, we have gathered a series of data relating to the capacity for reading, comprehension and writing, giving a series of tests to a sample of 26 subjects with the Down syndrome, some still integrated in the context of school, while others are no longer at school having already completed the planned study cycle. It is also our intention, at the conclusion of the research, to succeed in understanding which educational and/or didactic methods and which rehabilitative approaches have led to the best results in terms of development and conservation of basic educational skills. Although the sample on which we have gathered data is limited, we are already able to present some significant results pertaining to our research.
\end{abstract}

\section{Introduction}

Why a longitudinal research on the development and conservation of basic educational skills on a population with Down syndrome? First of all, because we are convinced that all children with the Down syndrome can achieve significant educational results as far as basic educational skills are concerned. The differences, sometimes considerable, that are found amongst pupils of the same age with the Down syndrome are only partly dependent on the clinical picture of the syndrome. It is often the early educational and rehabilitative intervention and the special didactic methodology used that make the real difference. Another reason is because we have been able to verify that when the basic educational skills have been learnt well, as well as lasting over time, they serve as a resource in the delicate passage from school to the world of work.

The longitudinal research that we present here is also born from the wish to verify whether the didactic methodologies experimented by us are in fact capable not only of developing as well as possible the learning capacity of children with the
Down syndrome that we have followed and supervised over time but whether the results are comparable - and to what extent - with the results achieved by children with typical development. To reach this objective, we have subjected our sample to a series of tests that are used to evaluate the capacity for reading, comprehension and writing of pupils with typical development.

The backward analysis of the educational and scholastic pathway of the sample, whose data we have gathered, has also offered us interesting information on which special didactic actions and/or educational processes seem to offer the best results in terms of development and conservation of basic educational skills. Since our sample is represented by subjects with the Down syndrome that are presently attending school and by subjects that, for some years, have been out of the system of school education, we have been able to gather significant data concerning the actual conservation of the reading, comprehension and writing capacity away from the school.

Because ours is a research on a sample of subjects with the Down syndrome that have attended the Italian school, which, as is well known, provides an educational model of the inclusive type, the data that emerges from the research also allows us to reflect on the effective efficiency of that model with regard to the development of learning the ability to read, comprehend and write on the part of pupils with the Down syndrome that have attended Italian schools.

\section{A look at the scientific literature on the development of the capacity to read, comprehend and write in presence of the Down syndrome}

In general, the study and research on the educational abilities of people with the Down syndrome (DS) does not have great prominence in the panorama of international scientific literature. An exception to this, if only in limited amount, is the research on the development of the capacity to read. However, it mainly concerns the research that has tried to verify whether the same elements are present 
in children with the DS, which give origin to the acquisition of the capacity to read in the typical population, such as for example, the phonological awareness, and phonological memory, as well as the memory of the letters [26]. The data point out that, despite the fact that people with DS have considerable difficulty in acquiring language, they are, in any case, able to develop a moderate capacity to read [14], [11], [14], [24]).

There is also research which explores the positive effects of the development of the capacity to read for children with DS; Learning to read, in fact, is shown to be an important factor of development for children with DS. The data demonstrates that learning to read promotes the development of language, the discriminative capacity of the visual and auditive type and the short-term memory [6], [17], [19].

However, there are few works that describe cases focused on the definition educational or rehabilitation methods for the teaching of reading (amongst these, the most recent are those of Lemons \& Fuchs [18]; Colognon, Cupples \& Wyver [9]), as well as studies that have evaluated the ability to read in larger groups of people with DS [7], [11], [30]. However, it is clear from the research that the educational choice of teaching a child with DS to read not only proves to be important but should be introduced into his educational and rehabilitation programme as early as possible [4].

Even while succeeding in learning to read, the pupils with DS, with a certain regularity, have difficulty with comprehension. In general, in the presence of a good capacity to read words compared to non/words (words without sense), they have a reduced ability to comprehend the text [1]. Even the good readers show a discrepancy between the recognition of the words and the comprehension while reading [8], [13], [14], [22]. Nash and Heath [20], for example, have compared the profile of persons with DS aged between 11-19 years with that of persons with typical development, discovering that the comprehension in reading of those with DS was at least 12 months under the typical level (compared to normal population of the same age) while reading single words. In general, the difficulties that are noticed in pupils with DS are comparable to those demonstrated by pupils of typical development with poor comprehension [22]. However, the longitudinal studies demonstrate that, in spite of the low level of progress in the development of the capacity to comprehend, significant improvements in maturity have nonetheless been noted [20], [30].

If the capacity for reading and comprehension of children with DS has been the subject of a great deal of research, the same thing cannot be said of the capacity for writing. The phenotype of the DS involves difficulties in motor functions [10], [31]. Therefore, it is generally accepted that motor difficulties are more present in children and adults with SD and, for that reason, the negative effect on the development of all the capacities related to it are considered inevitable, including the ability to write. The motor problems that lead to difficulties in the acquisition of writing are associated with hypotonia, ligamentous laxity, loss of control of posture, balance difficulty. Above all, it is the difficulty in performing tasks involving movement that are decisive in limiting the learning of writing [25]. Knowing how to write, in fact, is the result of a long process which involves motor, linguistic and visualspatial tasks, as well as perception [23] the result of which is anything but taken for granted, especially in the presence of DS [23]. A recent study has carried out an evaluation of the handwriting of adults with DS [28]. It has shown that, in particular, the deficiencies in organization of space affect the quality of writing. In general, the studies that have investigated the capacity for writing in children and adults with DS have concluded that their writing capacities are often simple, like succeeding in writing the shopping list [27], [29]. The longitudinal study of Turner and Alborz [29] found that, at 21 years of age, $23 \%$ of the sample analyzed had reached levels of reading, writing and calculating ability comparable to a pupil with typical development of 11 years, while $50 \%$ of the sample had reached capacities comparable to a level of 7 years, and $25 \%$ to a level of 5 years. An Italian study in 2000 [12] pointed out that $11 \%$ of the pupils in the first year of the Secondary School were not able to write, while $68 \%$ wrote single words autonomously, $39 \%$ short sentences and $10 \%$ brief compositions. In general, even if there is a growing awareness of the potentiality of alphabetization of children with DS, we can affirm that we do not know enough about their capacity for writing [28].

\section{Method of investigation}

We have given, to a sample of 26 subjects with DS, a series of tests, with which we have analyzed their capacity for reading, comprehension and writing.

The sample, distributed throughout the national territory and equally divided between male and female, has been identified choosing persons with DS that, in the first $6 / 7$ years of life, have performed an intensive work of rehabilitation at home. The activities carried out were: tactile, vestibular and motor stimulations (belly crawling, crawling on hands and knees, walking, running), fine use of hands (tactile boxes, red words, home book, alphabet material, fine use of hands with pre-handwriting and introduction to handwriting, intellectual programme, stimulation of hearing. The programme of rehabilitation began for all of the sample between 23 months after birth and 8-9 months. The ages taken 
into consideration range from 9 to 30 years. The sample was divided between participants who are outside the school system and those still attending school.

We met with considerable difficulty in identifying our sample. Our efforts to obtain statistically significant data imposed several constraints on evaluations and choices of the sample, primarily, the starting level of intellect (it was not possible to have this information for everyone, as it was not always available or up to date). We decided to proceed by choosing those who were capable of performing the tasks planned in the tests, in other words, those that showed the ability to read and write (regardless of the level reached). The presence of an intellectual disability, especially regarding the greater or lesser gravity of the same, creates such a disharmony amongst subjects with DS that it is difficult to have a homogeneous sample. The decision to take as part of the sample only those that were capable of being evaluated with the tests and reject those that could not be evaluated with the tests, seemed a criteria sufficiently valid, considering that we used tests that are normally used for pupils with typical development. Furthermore, we decided, in the cases where it was possible, to make video recordings of those taking the tests. Regarding the tests we used for the evaluation of the capacity for reading, comprehension and writing, our choice fell on the test protocol in use in Italy for the evaluation of learning difficulties in the Italian school population. In specific terms: MT-3 clinical trial, a series of tests for the evaluation of the ability to read and comprehend for the primary school and the first year of secondary school, Cesare Cornoldi \& Barbara Carretti, Giunti OS, 2016 Florence.

DDE-2 Tests for the Evaluation of Dyslexia and Evolutionary Dysorthography-2 [33].

The results obtained were evaluated according to the test criteria. It was decided to accept a standard deviation range from -0.99 to $+0.99 \mathrm{DS}$.

Beyond this range the school class level changes. Each performance was evaluated according to the mistakes the child could make, e.g. the average of the errors of the typical Italian student population was compared with the average of the errors of each participant in the research.

The average of the errors of the typical Italian student population allows a standard deviation that ranges from -0.99 to +0.99 .

The school results obtained are evaluated according to the sufficiency criterion, so when the test performance reaches $-06 \mathrm{DS}$, at this point the school class is identified.

The correlation of the results also takes into account the IQ, assessed by rehabilitation facilities. Parents, moreover, submit a structured questionnaire [32] to reconstruct the rehabilitation, school and autonomy pathways in life.
For every test used we divided the sample into two categories: participants who still attend school and participants who have left.

Results are organized in order to show the level reached by participants who still attend school and the level retained by those who have left school. We tested the sample with the three tests shown before based on the typical Italian student population to determine what level the sample has achieved compared to the abilities of typical Italian students.

We have collected data on the following:

Reading, comprehension, compared to the typical Italian student population (MT3)

Words Reading, velocity compared to the typical Italian student population.

Words Reading, errors compared to the typical Italian student population.

No-Words Reading, velocity compared to the typical Italian student population.

No-Words Reading, errors compared to the typical Italian population.

Homophones Reading Comprehension, compared to the normal Italian population. For example: suite - sweet.

Homophones Reading Spelling Correction, compared to the normal Italian student population. For example: lake - l'ake.

Words Writing (Dysorthography) compared to the normal Italian student population.

- No-Words Writing (Dysorthography) compared to the normal Italian student population.

Sentence Writing (Dysorthography).

The MT3 test was given in the form of successive tasks, each time looking for results in the statistical medium of sufficiency and identifying the class level (in giving the test, we looked for a sufficient performance contained within -06 DS, after which the school class level was identified). For the comprehension test we asked for a double reading of the text, in silence or aloud according to the preference of the individual. Each comprehension question was read more than once, and the people were helped to find the paragraph of the text that the question referred to. This required a great expenditure of mental energy and attentiveness on the part of the subjects involved (in many cases we were surprised at the length of time of attentiveness and the capacity for concentration on the task). In 3 cases, we had to rewrite the test with larger writing because of a visual impairment. The tasks of the DDE-2 tests were given according to protocol.

\section{Results}

All the test results show that $70 \%$ of the partial sample has reached a level higher than the 2 nd grade of the primary school. 
The results show that people who have completed school have maintained good performances and autonomy in reading and writing.

These results describe a positive trend that we hope we will be able to confirm at the conclusion of the research, based on the data of the whole sample, composed of 100 people with Down syndrome. Here, in detail, is what emerged from our sample of 26 people with Down syndrome:

Reading comprehension compared to the normal Italian student population:

- Inside school system, 12 participants out of 17 beyond PS 2nd grade.

- Outside school system, 8 participants out of 9 beyond PS 2nd grade.

Word Reading, velocity compared to the normal Italian student population:

- Inside school system, 13 participants out of 17 beyond PS 2nd grade.

- Outside school system, 7 participants out of 9 beyond PS 2nd grade.

Word Reading, errors compared to the normal Italian student population:

- Inside school system, 14 participants out of 17 beyond PS 2nd grade.

- Outside school system, 9 participants out of 9 beyond PS 2nd grade.

No-Word Reading, velocity compared to the normal Italian student population:

- Inside school system, 14 participants out of 17 beyond PS 2nd grade.

- Outside school system, 8 participants out of 9 beyond PS 2nd grade.

No-Word Reading, errors compared to the normal Italian student population:

- Inside school system, 14 participants out of 17 beyond PS 2nd grade.

- Outside school system, 8 participants out of 9 beyond SS 2nd grade.

Homophones Reading Comprehension compared to the normal Italian student population:

- Inside school system, 14 participants out of 17 beyond PS 2nd grade.

- Outside school system, 8 participants out of 9 beyond PS 2nd grade.

Homophones Correction compared to normal Italian student population:

- Inside school system, 11 participants out of 17 beyond PS 2nd grade.

- Outside school system, 7 participants out of 9 beyond PS 2nd grade.

Word Writing (dysortography) compared to the normal Italian student population:

- Inside school system, 14 participants out of 17 beyond PS 2nd grade.

- Outside school system, 7 participants out of 9 beyond PS 2nd grade.

No-Word Writing compared to the normal Italian student population.
- Inside school system, 15 participants out of 17 beyond PS 2nd grade.

- Outside school system 9 participants out of 9 beyond PS 2nd grade.

Sentence Writing:

- Inside school system, 10 participants out of 17 beyond PS 2nd grade.

- Outside school system, 9 participants out of 9 beyond PS 2nd grade.

\section{Considerations made after collection of the first results}

We had the opportunity to reconstruct the history of school learning of the various subjects evaluated, both by looking at school exercise books and by collecting information regarding the didactic practices used with them at school. We also gathered useful information to understand whether, as well as schoolwork, other methodologies of work in the home, after school, were adopted, and whether interventions of rehabilitation were made and what type.

On a first analysis of the data collected by us, an interesting picture emerges, which shows that persons with DS that have followed an educational programme at school and at home with the involvement of the parents, are those that demonstrate the achievement of a school level situated in the middle range of pupils with typical development.

Another fact we have established is that only those persons with DS who, in the first $6 / 7$ years of life, have followed an intensive programme of the educational and rehabilitative type, manage to reach the highest level of scholastic competence. Included amongst these are the ones that maintain the best reading, comprehension and writing ability after school, even several years after they finish attending school. To confirm this fact, we have ascertained that all those that passed the most difficult reading and comprehension test had the experience of an early stimulation in reading. It appears evident that the first $6 / 7$ years in the life of a child with DS are fundamental to construct the architecture necessary for learning reading and writing.

As far as special didactic practices are concerned, we have established that those who had the best results in reading and writing were the ones who used an alphabet book. The use of this didactic instrument, based on what we observed during the test procedure, has a beneficial effect in reducing spelling errors. We assume that the use of the alphabet book has reduced dysorthography, since such a didactic instrument means that the pupil who uses it for reading and writing has to learn letter by letter and this permits a better organization of the perception of sounds (phonemes), of letters and of 
shapes, as well as the sounds deriving from the putting together of letters (morphemes), enabling a better processing of the transcription/transformation of sounds/signs and vice versa.

Regarding the capacity for comprehension, while giving the test we realized that such a capacity could have been improved only if it had been exercised to a greater extent. Many of those to whom we gave the test told us they had a real passion for reading, and that they practise every day reading books of various nature (detective stories and adventure novels in particular).

\section{Conclusion}

The data that we have collected are part of a reduced sample with regard to the research that we are carrying out. The collection and analysis of all the data concerning the entire research sample will certainly give us further information concerning the objectives of this study.

However, we have already been able to verify that the development and maintaining of the basic scholastic competence of persons with DS prove to be closely related to pre-scholastic educational work, carried out with the collaboration of the parents. In particular, we have been able to ascertain that it is those persons with the Down syndrome who have done an intensive work of rehabilitation at home, starting from the first year of life, who obtain the best results concerning the learning of basic scholastic skills as well as the maintenance of that learning. This data leads us to consider it fundamental to develop and share amongst experts an educational/rehabilitative work protocol, to be put into effect early in life and to share with the family, and that indicates which are the interventions that can help children with DS to best develop the prerequisites of school learning and which educational activities can help them develop and maintain the basic scholastic skills. Such a work protocol is part of our future plans, as a follow up to the ten-year experience that we have been able to develop in the field.

\section{References}

[1] Boudreau, D. (2002). "Literacy skills in children and adolescents with Down syndrome". Reading and writing an interdisciplinary journal, 15, pp. 497-525.

[2] Buckley, S.J, Bird, G., Sacks, B., Archer, T., (2006). "A comparison of mainstream and special education for teenagers with Down syndrome: Implications for parents and teachers". Down Syndrome Research and Practice, 9 (3), pp. 54-67.

[3] Buckley, S. (1985). Attaining basic educational skills: Reading, writing and numbers. In: D. Lane \& B.
Stratford. (eds), Current approaches to Down syndrome, pp. 268-279. New York: Praeger.

[4] Buckley, S., Bird, G., Byrne, A. (1996). Reading acquisition by young children. In: B. Strafford \& P. Gunn (eds). New approaches to Down syndrome, pp. 268-279, London: Cassell.

[5] Byrne, A, (1997). The development of reading skills in children with Down syndrome. Unpublished PhD thesis. University of Portsmouth UK.

[6] Byrne, A., Buckley, S., Macdonald, J. \& Bird, G. (1995). "Investigating the literacy, language and memory skills of children with Down's syndrome". Down's syndrome: Research and practice, 3, pp. 53-58.

[7] Byrne, A., Macdonald, J. \& Buckley, S. (2002). "Reading, language and memory skills: A comparative longitudinal study of children with Down syndrome and their mainstream peers" British Journal Educational Psychology, 72, pp. 513-529.

[8] Cardoso-Martins, C., Peterson, R., Olsen, R. \& Pennington, B. (2009). "Component reading skills". Down syndrome. Reading and Writing, 22, pp. 277-292.

[9] Colognon, K., Cupples, L. \& Wyver, S. (2011). "Effects of targeted reading instructions of phonological awareness and phonic decoding in children with Down syndrome". American Journal of Intellectual and Developmental Disabilities, 16, pp. 111-129.

[10] Davis, A. S., (2008). "Children with Down syndrome: Implications for assessment and intervention in the school". School Psychology Quarterly, 23, pp. 271281.

[11] Fletcher, H. \& Buckley, S. (2002). "Phonological awareness in children with Down syndrome".Down Syndrome Research and Practice, 8, pp. 11-18.

[12] Gherardini, P., Nocera, S. (2000). L'integrazione scolastica delle persone Down. Una ricerca sugli indicatori di qualità in Italia. (The inclusion in mainstream schools of persons with Down Syndrome.), Trento (Italy): Centro Studi Erichson.

[13] Groen, M., Laws, G., Nation, K. \& Bishop, D. V. M. (2006). "A case of exceptional reading accuracy in a child with Down syndrome: Underlying skills and the relation to reading comprehension". Cognitive neuropsychology, 23, pp. 1190- 1214.

[14] Kay-Raining Bird, E., Cleave, P., \& McConnell, L. (2000). "Reading and phonological awareness in children with Down syndrome: A longitudinal study". American Journal of Speech-Language Pathology, 9, pp. 319-330.

[15] Laws, G., Briscoe, J., Ang, S. Y., Brown, H., Hermena, E., \& Kapikian, A. (2014). "Receptive vocabulary and semantic knowledge in children with SLI and children with Down syndrome". Child Neuropsychology. A Journal on Normal and Abnormal Development in Childhood and Adolescence, 21:4, pp. 490-508. 
[16] Laws, G., Brown, H., Main, E. (2016). "Reading comprehension in children with Down syndrome".Reading and writing, 29, pp. 21- 45 .

[17] Laws, G. (2010). "Reading as an intervention for vocabulary, short term memory and speech development of school-aged children with Down syndrome". Advances in Child Development and Behavior, 39, pp. 131-162.

[18] Lemons, C. J., Fuchs, D. (2010). "Phonological awareness of children with Down syndrome: its role in learning to read and the effectiveness of related interventions". Research in Developmental Disabilities, 31:2, pp. 316-330.

[19] Naess, K. A. B., Lervag, A., Lyster, S. A. H., Hulme, C. (2015). "Longitudinal relationships betweenlanguage and verbal short-term memory skills in children with Down syndrome". Journal of Experimental Child Psychology, 135, pp. 43-55.

[20] Nash, H. \& Heath, J. (2011). "The role of vocabulary, working memory and inference making ability in reading comprehension in Down syndrome". Research in Developmental Disabilities, 32, pp. 1782-1792.

[21] Roch, M., Jarrod, C., (2008). "A comparison between word and nonword reading in Down syndrome: The role of phonological awareness". Journal of Communication Disorders, 41, pp. 305-318.

[22] Roch, M., \& Levorato, M. C. (2009). "Simple View of Reading in Down's syndrome. The role of listening comprehension and reading skills". International Journal of Language and Communication Disorders, 44, pp. 206-223.

[23] Sangalli, A. L., Lascioli, A., Lascioli, A. (2018). Dysgraphia, educational interventions and didactic implications: from prevention to intervention. In: Education Applications and Developments III, edited by Mafalda Carmo (WIARS) Portugal: Inscience Press, In press.

[24] Snowling, M. J., Hulme, C., \& Mercer, R. C. (2002). "A deficit in rime awareness in children with Down syndrome". Reading and Writing, 15, pp. 471-495.

[25] Spanò, M., Mercuri, E., Randò, T., Pantò, T., Gagliano, A., Henderson, S., \& Guzzetta, F. (1999). "Motor and perceptual-motor competence in children with Down syndrome. Variation in performance with age". European Journal of Pediatric neurology, 3, pp. 7-13.

[26] Swan, D., \& Goswani, U. (1997). "Phonological awareness defects in developmental dyslexia and the phonological representations hypothesis". Journal of Experimental Child Psychology, 60, pp. 334- 353.

[27] Trenholm, B., \& Mirenda, P. (2006). "Home and community literacy experiences of individuals with Down syndrome". Down Syndrome Research and Practice, 10, pp. 30-40.

[28] Tsao, R., Fartoukh, M., Barbier, M. L. (2011). "Handwriting in adults with Down syndrome". Journal of Intellectual and Developmental Disability, 36:1, pp. 20-26.
[29] Turner, S., Alborz, A., (2003). "Academic attainments of children with Down's syndrome. A longitudinal study". British Journal of Educational Psychology, 73, pp. 563-583.

[30] Verrucci, L., Menghini, D., \& Vicari, L. (2006). "Reading skills and phonological awareness acquisition in Down syndrome". Journal of Intellectual Disability Research, 50, pp. 477-491.

[31] Vicari, S., (2006). "Motor development and neuropsychological patterns in persons with Down syndrome”. Behavior Genetics, 36, pp. 355-364.

[32] Sparrow S.S., Cicchetti V.D., Balla A.D., (2005) "Vineland adaptive behavior scales". 2nd edition, American Guidance Service, Circle Pines, MN.

[33] Sartori G., Job R., Tressoldi P.E., (2007), Giunti OS, Florence. 